\title{
Do agostic interactions play a role in the stabilization of the nido structure of [(PPh3)2RhSB9H10]?
}

Jonathan Bould (Institute of Inorganic Chemistry of the AS CR, v.v.i., 25068 HusinecRez, Czech Republic)

Ramón Macías (ISQCH, Universidad de Zaragoza-CSIC, C/Pedro Cerbuna 12, 50009 Zaragoza, Spain)

A recent perspective published in Chemical Communications deals with the impact of Wade's Rules on the perceptions of bonding within clusters in which the framework is bound through delocalized bonding. A particular case of the structure of [L2RhSB9H10] ( $\mathrm{L} 1 / 4 \mathrm{PPh} 31$, or $\mathrm{L} 2 \mathrm{1} / 4 \mathrm{PPh} 2 \mathrm{CH} 2 \mathrm{CH} 2 \mathrm{Ph} 2 \mathrm{P}$, dppe) cluster compounds and their associated electron counts, was prominently described to illustrate an instance where, using Wade's Rules, a seemingly anomalous electron-count encouraged the author and his co-workers to look more deeply at the cluster compound in order to resolve the anomaly. The proposed inconsistency arose because the rhodathiaborane cluster 1 was taken to possess 12 skeletal-electron bonding pairs: nine pairs from the nine $\mathrm{BH}$ vertices, two pairs from $S$ and one pair in total from the bridging $\mathrm{mH}$ atom plus the $\{\mathrm{L} 2 \mathrm{Rh}\}$, rhodium (I) moiety, and this is one skeletal-electron bonding pair short of the 13 required by the application of Wade's Rules for a nido 11-vertex cluster geometry. Welch et al. posited that the observed nido structure is stabilized because the cluster receives an extra pair of electrons via two, one-electron agostic interactions with the rhodium centre from two phenyl ortho-hydrogen atoms on the phosphine ligands. The agostic interactions proposed to be observed in the solid state were not evident from NMR spectroscopic examination of the compound in solution. However, evidence of the effect was adduced from experiments where the bridging hydrogen atom in [(PPh3)2RhSB9H10] or [(dppe)2RhSB9H10] compounds were removed as a proton, using a strong base. The resulting anionic compounds, e.g. [(PPh3)2RhSB9H9]_ 2, now exhibit the classical closo structure expected for an 11-vertex metallaborane polyhedron with 12 skeletal electron pairs. Crucially, although only a proton, and thus no electron, was removed from the nido cluster, the "loss" of one skeletalelectron bonding-pair of electrons implied by the nido to closo structural change was attributed to the "turning off" of the agnostic interactions. It was consequently taken as evidence of the proposed agostic interactions, of their electron donating effect, and, by an implicit corollary, a confirmation that the $\mathrm{L} 2 \mathrm{RhSB} 9 \mathrm{H} 10$ clusters do indeed require 13 skeletal electron pairs in order to achieve a nido structure. Discrepancies between electroncounting rules and structure are common among polyhedral molecules that incorporate $\mathrm{C} 2 \mathrm{v}$ fragments such as $\{\mathrm{L} 2 \mathrm{Rh}\}$ or $\{\mathrm{L} 2 \mathrm{Pt}\}$. This phenomenon has long been recognized and rationalized, and it has been dealt with in the literature several times in the context of metallaborane chemistry, showing that metallaundecaborane clusters can be one or even two skeletal-electron bonding pairs short of those formally required by Wade's Rules. Briefly, this discrepancy arises because Wade's Rules, when applied to binary boron hydrides with endoterminal hydrogen atoms (e.g. in a $\mathrm{BH} 2$ moiety in [B11H14]), enter the endo-terminal hydrogen atoms and their associated electrons into the electron count even though the electrons and orbitals in the exo- and endo-hydrogen atoms are not involved directly in bonding to the other cluster vertices. When a squareplanar 16-electron metal-ligand moiety such as $\{\mathrm{L} 2 \mathrm{Rh}\}$ replaces the $\{\mathrm{H} 2 \mathrm{~B}\}$ moiety, the metal still contributes two orbitals and one electron to the cluster bonding but it lacks the extra hydrogen atom and thus the two electrons involved in the endo $\mathrm{BeH}$ vector that are formally entered into the cluster electron count in the case of $\{\mathrm{H} 2 \mathrm{~B}\}$ and it therefore appears to be one skeletal electron pair short of that required by the rules. 
In otherwords,Wade's rules only count the electrons donated to the cluster not the orbital contribution, and they assume that transition metal centres adhere to the 18electron rule. If the metal vertex uses three orbitals in the cluster bonding, then 12 of the 18 valence electrons available at the metal centre are not involved in cluster bonding. These premises have been generalized to give the number of skeletal electrons per metal vertex as $\left(v p x_{-} 12\right)$, where $v$ is the number of valence electrons of the metal and $x$ the number of electrons donated by the exo-polyhedral ligands. Thus, the $\{R h L 2\}$ vertex in compound 1 (and its dppe analogue) contributes one electron to the skeletal cluster bonding (i.e. 9 p 4 12 1/4 1). From the perspective of the metal centre, the 11-vertex rhodathiaboranes, [L2RhSB9H10], can be regarded as 16-electron pseudosquare-planar $\mathrm{Rh}(\mathrm{I})$ complexes. Thus, the metal vertex, $\{\mathrm{RhL} 2\}$, does not adhere to the 18-electron rule. It is not surprising, therefore, that the electron count of these species does not agree withWade's rules. Now that the interpretation invoking agostic interactions to supply "missing" electrons has appeared in a primary journal such as Chemical Communications and also in an important reference source such as Comprehensive Organometallic Chemistry, we have been prompted to look for evidence of agostic bonding using DFT calculation. DFT calculations on 1 to look for evidence of a close approach of the ortho-phenyl hydrogen atoms to the rhodium centre were carried out using the B3PW91 level of theory with the $6-31 \mathrm{G}^{*}$ basis set for the main group atoms and the SDD basis set for $\mathrm{Rh}$. This level of calculation should be more than sufficient to determine the presence of what must be a fairly strong level of interaction with the rhodium centre if the hydrogen atoms are to share a significant level of electron density with the nidocluster. Analogous calculations at this level have been carried out previously to distinguish between agostic and non-agostic interactions. The calculation results in $\mathrm{Rh} / \mathrm{H}$ (ortho) distances of 2.910 and $2.935 \mathrm{~A}$. In the reported X-ray structure of , these distances are 2.955 and 3.007 A. In the X-ray refinement all phenyleH distances were placed in calculated positions, i.e. 0.95 _A. However, if the $\mathrm{CeH}$ (ortho) bond distance in the X-ray structure are normalized to 1.09 A, closer to distances based on more realistic neutron diffraction data, then the measured $\mathrm{Rh} / \mathrm{H}$ lengths are now 2.912 and 2.935 _A respectively. Thus, the calculated geometry reproduces the measured structure very closely. These $\mathrm{Rh} / \mathrm{H}$ (ortho) distances are very long compared to other well defined agnostic interactions of $1.8 \mathrm{e} 2.4$ A. Examples of agostic interactions in metallacarbaborane clusters are available in the literature. For example, [(C8H13)-closo-IrC2B9H10 $(\mathrm{CH} 2) 2 \mathrm{C} 6 \mathrm{H} 4] \quad 3$ and $[(\mathrm{C} 5 \mathrm{H} 9)(\mathrm{CH} 3) 2$-closo-RhC2B9H9] 4, show clear evidence in both the solid state and in measured NMR parameters of agnostic bonding, and these were not been proposed to play any role in the cluster bonding. $\mathrm{H}$-metal distances in these compounds are at 1.77(5) and 2.09(4) _A respectively, significantly shorter than the $\mathrm{Rh} / \mathrm{H}$ (ortho) distances in compound 1. A further indication of a significant agostic interaction would be evident in the calculated phenyl $\mathrm{CeH}$ (ortho) bond distances in 1, with the distance increasing in line with the strength of the contact; indeed, the equivalent methyl $\mathrm{CeH}$ (agostic) measured distance in 3 is 1.14(5) _ A compared to the measured non-agostic $\mathrm{CeH}$ distances of $0.86 \mathrm{e} 0.99$ A. The relevant calculated, proposed agostic, $\mathrm{CeH}$ distances in 1 are 1.088 and $1.08 \overline{6}$ _A with the remaining $\mathrm{CeH}$ (ortho) distances ranging from ca. 1.085 to 1.087 A. These are not appreciably different and therefore show no perceptible agostic interaction. The nido-1 to closo-2 transformation on removal of the bridging hydrogen atom in 1 may be explained in terms of the stabilization effect of the BeHeB hydrogen atom rather than invoking the "turning off" of agostic interactions. The removal of the hydrogen atom (as a proton) on the open face allows the cluster to close up. Geometry minimization at the RB3PW91/6-31 $\mathrm{pG}^{*}$ level on both the nido and closo conformations of [(PH3)2RhSB9H9]_, with a phenyl for hydryl replacement for reasons of computational economy, indicate that the closo isomer is $11 \mathrm{kcal}$ mol_1 lower in energy than the nido isomer. This is clearly a strong indication that the putative agostic interactions play no decisive role in stabilizing the geometry of the nido form compared to the closo. 
The energy difference between the two isomers reflects the stabilization inherent in going from the asymmetric nido $\mathrm{C} 1$ to closo idealized Cs symmetry with driving force for the closure being provided by the negative charge on the open face being taken up by the inherently Lewis-acidic 16-electron metal centre. Finally, it may be noted that a converse energy difference is observed in PH3 models for 1 itself, as depicted in. The nido cage is $11 \mathrm{kcal}$ mol_1 more stable than its isonido isomer, which exhibits a pseudo-square open face and a hydride ligand trans to the sulphur vertex. The nido cluster is inherently more stable than its isonido isomer. Thus, without any possible contribution in these $\mathrm{PH} 3$ models from agostic interactions from $\mathrm{P}$-aryl hydrogen atoms or from other molecules, it is clear that the isomer with a BeHeB bridging hydrogen atom on the pentagonal face of the nido-cluster is more stable in this 11-vertex rhodathiaborane system.

DFT calculation together with a consideration of the measured interatomic distances in [(PPh3)2RhSB9H10] 1 offers no evidence for the proposition that the nido, rather than the closo structure of 1, results from cluster bonding electrons being donated by two agostic interactions. As mentioned in the introduction, 11-vertex metallaundecaborane clusters containing $\{L 2 R h\}$ moieties do not require the 13 skeletal-electron-pairs predicted by a simple application of Wade's Rules to attain a nido structure. We suggest that, rather than being an example ofWade's Rules stimulating a deeper understanding of this system, the case in point is actually an example of the overapplication ofWade's Rules, an attempt to fit a cluster electron count into a case where the rules do not apply in a straight-forward manner.

Acknowledgements: JB acknowledges the support of the Grant Agency of the Czech Republic, Grant No. P207/11/1577.

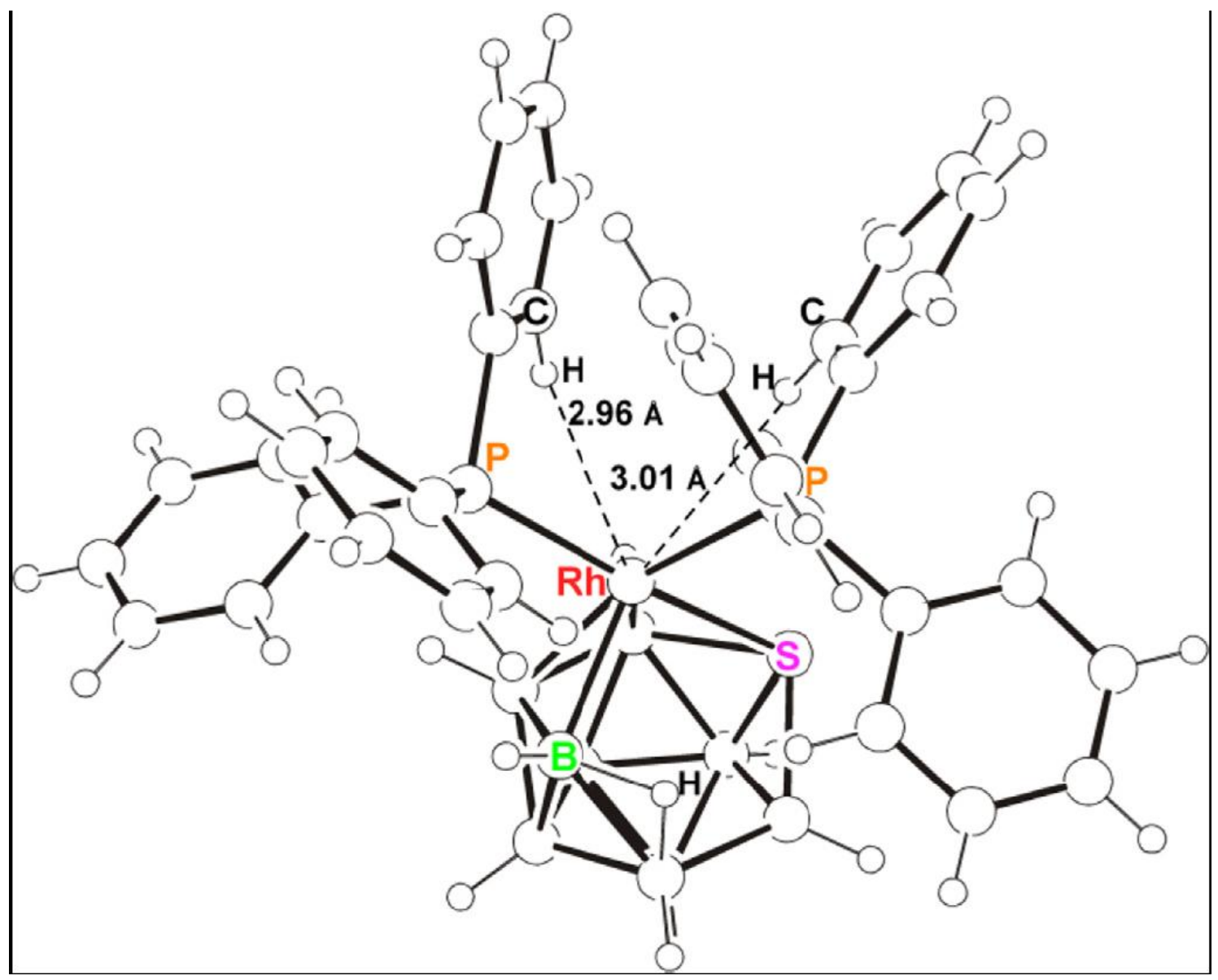

Fig. 1. Drawing of [8,8-(PPh3)2-nido-8,7-RhSB9H10] 1 showing the closest phenyl hydrogen atom distances (in _A) to the rhodium centre. Atomic coordinates taken from single-crystal X-ray data. 


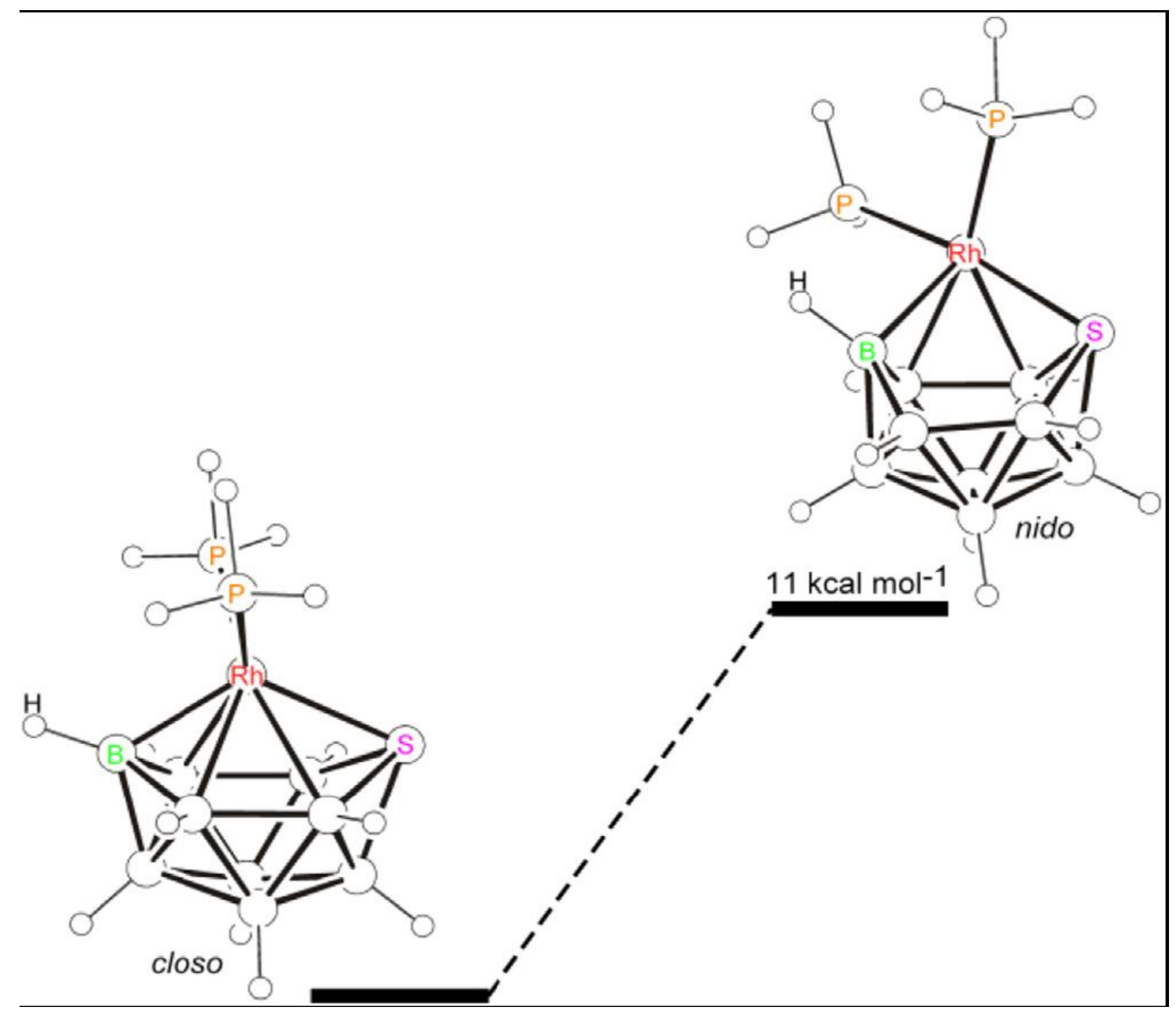

Fig. 2. Energy comparison for DFT optimized isomeric closo and nido structures of the PH3 model anion, [(PH3)2RhSB9H9]_ 2. 


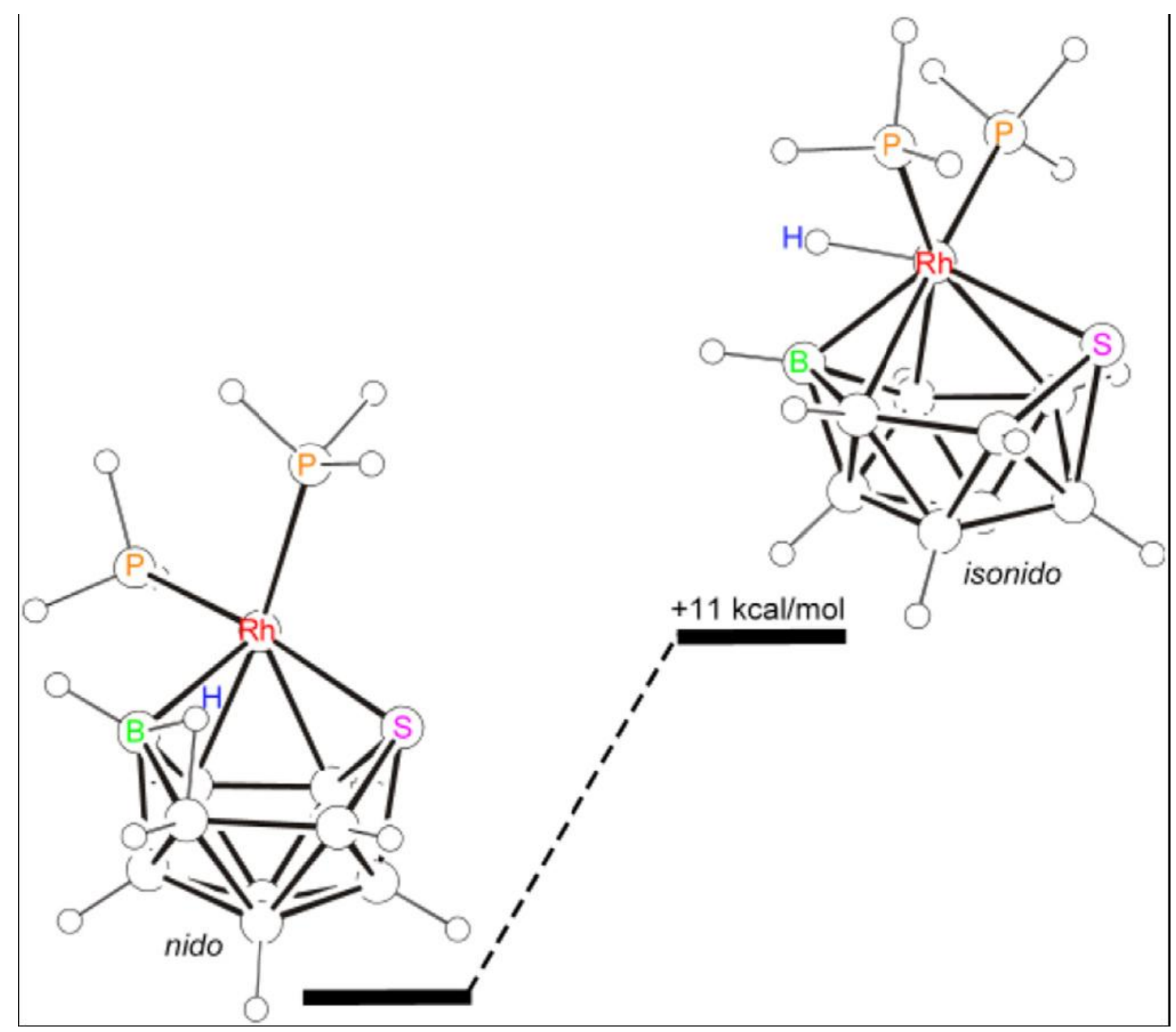

Fig. 3. Energy comparison for DFT optimized isomeric nido and isonido structures of the neutral $\mathrm{PH} 3$ model of 1, [(PH3)2RhSB9H10]. 